\title{
Muon Performance with CMS detector in Run2 of LHC
}

\section{Kyeongpil Lee ${ }^{* \dagger}$}

Seoul National University (KR)

E-mail: kplee@cern.ch

The Compact Muon Solenoid (CMS) detector is one of the two multi-purpose experiments at the Large Hadron Collider (LHC) and has a broad physics program. Many aspects of this program depend on our ability to trigger, reconstruction and identify events with final state muons in a wide range of momenta, from few $\mathrm{GeV}$ to the $\mathrm{TeV}$ scale. In this talk we present the full process of muon reconstruction in CMS, both offline and online. The identification and isolation strategies to discriminate prompt muons from background, and their performance with $13 \mathrm{TeV}$ data collected with the CMS experiment.

The 39th International Conference on High Energy Physics (ICHEP2018)

4-11 July, 2018

Seoul, Korea

* Speaker.

${ }^{\dagger}$ CMS Collaboration 


\section{Introduction}

Muons play an important role in many LHC physics analyses from precision measurements to new physics searches due to their clean signal for a wide range of momentum. This talk introduces how the offline and online muons are reconstructed and what their performance is with the CMS detector for Run2 of LHC.

\section{Offline reconstruction}

\subsection{Reconstruction procedure}

The reconstruction of muons in CMS starts from two kinds of tracks independently reconstructed in different sub-detectors. The tracker track is made in the inner tracker system by running a sequence of tracking algorithms with Kalman filter technique. The other one is the standalone track reconstructed with Kalman filter technique using the information from the muon system.

The next step is to build muon tracks by combining the information from each sub-detector using two different approaches. The outside-in (OI) approach makes the global muon by matching the standalone tracks to the tracker tracks. This approach is powerful to suppress the misidentification rate and have better resolution in high- $P_{T}$ region. From the inside-out (IO), approach, tracker muons are reconstructed by matching tracker tracks to segments in the muon system. This approach allows more chance to be reconstructed for low- $P_{T}$ muons.

\subsection{Identification procedure}

After the muons are reconstructed, muon identification is necessary according to its usage in each physics analysis. By the combination of several requirements on the muon types and the variables related to the muon reconstruction quality, several standard sets of identification requirements (ID) are made and widely used in CMS from loose one to tight one. In addition, special IDs for B physics and high- $P_{T}$ muons are also developed with several tuning on requirements in ID.

Muon isolation is also commonly used to distinguish prompt muons from the ones inside of jets. Track-based isolation uses the sum of reconstructed track in a $\Delta \mathrm{R}$ cone near a muon, which leads high efficiency and small dependence on pileup. The other one is Particle-Flow (PF) based isolation constructed by the sum of charged and neutral particles from PF algorithm. This isolation has better suppression power for the muons from jets than track-based isolation. More details can be found in [1].

\subsection{Identification performance}

Fig. 1 shows the performance of muon ID and isolation of the data collected in 2017. Left plot is the comparison of tight ID efficiency between data and Monte-Carlo (MC) simulation as a function of muon $\eta$. Overall data efficiency is about $97 \%$ and this is well described by the simulation within $1-3 \%$. Efficiency drop near $\eta=0.3$ is due to the detector geometry.

On the other hand, right plot shows PF based isolation efficiency with respect to the muon satisfying tight ID. The overall efficiency is about $96 \%$ and data and MC agree very well within $0.5 \%$. More performance plots can be found in [2]. 

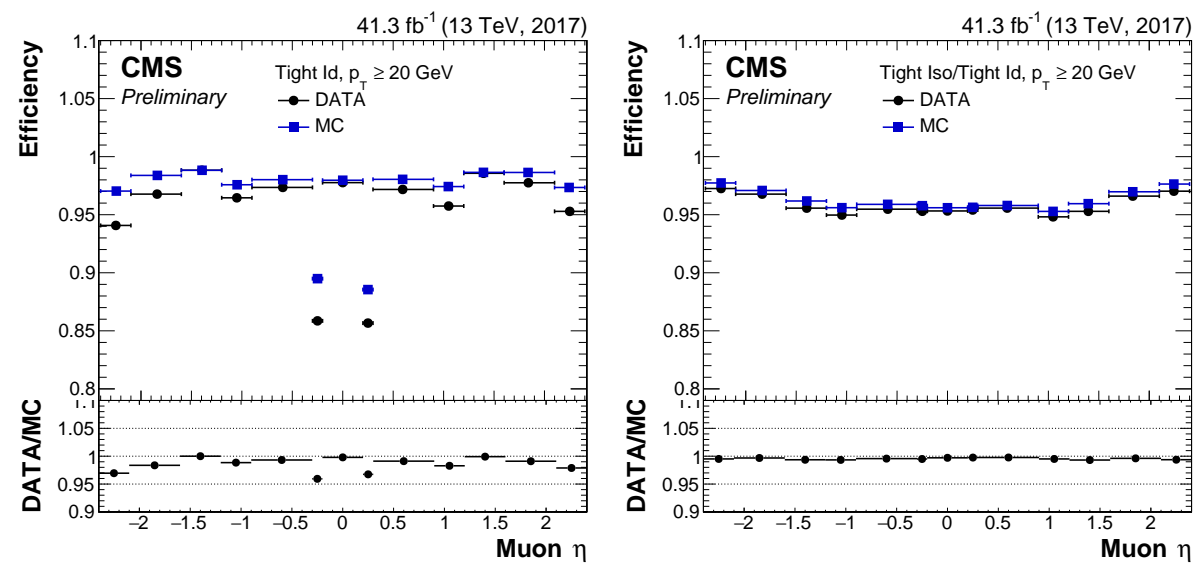

Figure 1: Comparison of efficiency between 2017 data and MC for Tight ID (left) and PF based isolation (right) as a function of muon $\eta$.

\section{Online reconstruction}

\subsection{CMS muon trigger system}

The online muon reconstruction starts from level-1 (L1) muon trigger which reconstructs trigger primitives using muon system information. If an event is accepted by L1 muon trigger, the event is moved to the muon high-level trigger (HLT) which is composed of 3 steps. First of all, L2 muons are reconstructed using the segments in muon system based on L1 seeds. In the second step, L3 muon reconstruction is performed by combining the inner track information with L1 or L2 muons. Finally, various filters on muon variables are applied to L3 muons and the event is accepted if L3 muons survive.

\subsection{L3 muon reconstruction}

During Run2, L3 muon reconstruction step has been evolved as it is the crucial part of muon HLT.

In the first 2 years of Run 2 (2015-16), two independent algorithms were used: Cascade with L2 seed and TkMu with L1 seed. Cascade algorithm consists of 2 OI and 1 IO algorithms, which run sequentially from the fastest to the slowest one to save the timing. The TkMu algorithm is HLT version of tracker muon reconstruction with the optimization of the timing. The OR combination of two algorithms was used to exploit the compensate power to each other.

In 2017, both algorithms are replaced by a new algorithm so called "iterative L3", based on the iterative tracking algorithm, to reduce the duplication of muon reconstruction with better performance. The algorithm is made of 3 steps which run sequentially: outside-in step from L2 seed (OIFromL2), inside-out step from L2 seed (IOFromL2) and inside-out step from L1 seed (IOFromL1). Therefore, the algorithm covers both L1 seeded and L2 seeded track reconstructions.

In 2018, further improvement on iterative L3 algorithm has been carried out by generating more seeds in IOFromL2 step especially in overlap and endcap region, adding one more iterative tracking in IOFromL2 and IOFromL1 steps, and applying a simple ID filter. As a result, higher efficiency with lower rate has been achieved compared to the 2017 data taking. 


\subsection{Performance}

Fig. 2 shows the efficiencies of muon triggers with 2018 data with respect to the offline muons passing ID and isolation. Left (right) plot corresponds to the result for isolated (non-isolated) single muon trigger with $P_{T}>24$ (50) $\mathrm{GeV}$ requirement. In both cases, the efficiency in the endcap region has been significantly improved after the update. More performance plots are available in [3].
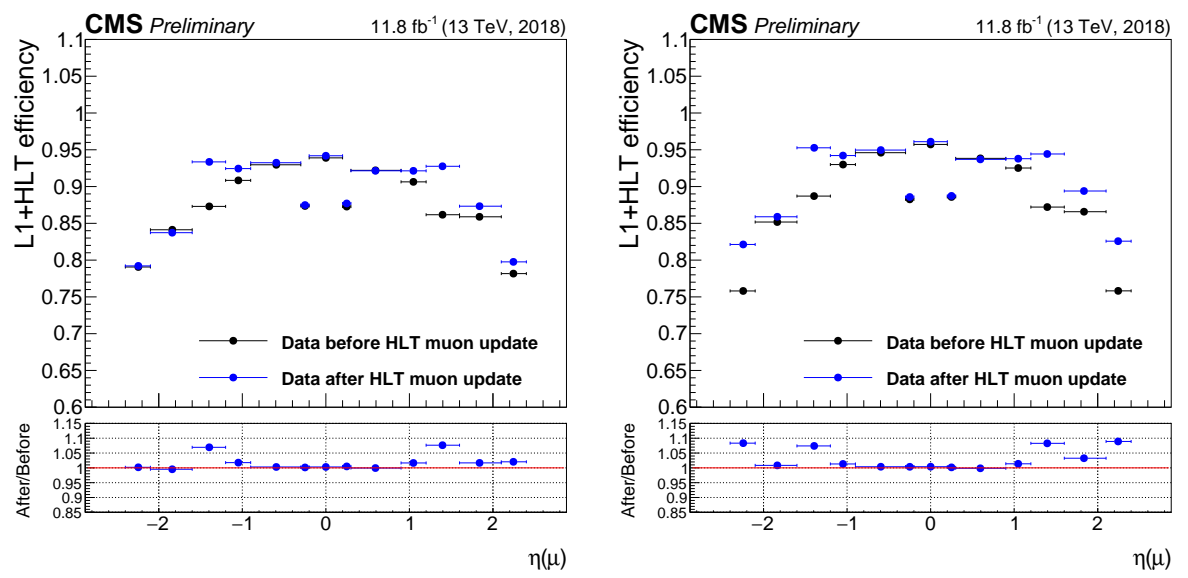

Figure 2: Efficiencies of isolated (left) and non-isolated (right) single muon triggers as a function of muon $\eta$.

\section{Summary}

The strategy for both online and offline muon reconstruction with the CMS detector in Run2 of LHC is presented. Due to the various upgrades in hardware and software parts during long shutdown1 and Run2, high performance for muon reconstruction and identification has been achieved with $97-100 \%, 96-98 \%$ and $90-92 \%$ efficiencies for ID, isolation and trigger respectively. This benefits all CMS Run2 analyses with muons.

\section{Acknowledgments}

Kyoengpil Lee is supported in part by the National Research Foundation of Korea (NRF) funded by the Korea government (NRF-2018R1A1A1A05077514 and NRF-2015R1A4A1042542).

\section{References}

[1] CMS Collaboration, Performance of the CMS muon detector and muon reconstruction with proton-proton collisions at $\sqrt{s}=13 \mathrm{TeV}$, JINST 13 (2018) P06015

[2] CMS Collaboration, Muon identification and isolation efficiencies with 2017 and 2018 data, CMS DP-2018/042

[3] CMS Collaboration, Muon HLT Performance with 2018 Data, CMS DP-2018/034 